\title{
Turán Type Inequalities for Jacobi-Dunkl Polynomials
}

\author{
Frej Chouchene* \\ Department of Mathematics, University of Sousse, Higher School of Sciences and Technology of Hammam Sousse, \\ 4011 Hammam Sousse, Tunisia \\ *Corresponding author: frej.chouchene@essths.u-sousse.tn
}

Received January 01, 2018; Revised April 02, 2018; Accepted May 20, 2018

\begin{abstract}
In this paper we establish Turán type inequalities for Jacobi-Dunkl polynomials. These complete an earlier result proven by G. Gasper in [1], for Jacobi polynomials.
\end{abstract}

Keywords: Jacobi polynomials, Jacobi-Dunkl polynomials, Turán type inequalities

2010 Mathematics Subject Classification: 33C45, 33C47, 42A05.

Cite This Article: Frej Chouchene, "Turán Type Inequalities for Jacobi-Dunkl Polynomials." Turkish Journal of Analysis and Number Theory, vol. 6, no. 3 (2018): 72-83. doi: 10.12691/tjant-6-3-2.

\section{Introduction}

The famous result of P. Turán [2], established in 1950, is the following inequality:

$$
\begin{aligned}
& \forall n \in \mathbb{N} \backslash\{0\}, \forall x \in[-1,1], \\
& \Delta_{n}(x):=P_{n}^{2}(x)-P_{n-1}(x) P_{n+1}(x) \geq 0,
\end{aligned}
$$

where $P_{n}(x)=\frac{1}{2^{n}} \sum_{k=0}^{n}\left(\begin{array}{l}n \\ k\end{array}\right)^{2}(x-1)^{n-k}(x+1)^{k}$, is the Legendre polynomial of degree $n$. This inequality has found much attention and several authors have deduced analogous results for other classical polynomials and special functions. For details we refer the interested reader to [1,3-17] and their references. There are many applications of these results, for example in numerical integration, information theory, economic theory and biophysics (see [18]).

Legendre polynomials belong to the class of Jacobi polynomials, which are studied in detail in $[19,20,21]$. For $\alpha>-1, \beta>-1, n \in \mathbb{N} \backslash\{0\}$ and $x \in[-1,1]$ let

$$
\Delta_{n}^{(\alpha, \beta)}(x):=\left(R_{n}^{(\alpha, \beta)}(x)\right)^{2}-R_{n-1}^{(\alpha, \beta)}(x) R_{n+1}^{(\alpha, \beta)}(x),
$$

where

$$
\begin{aligned}
& R_{n}^{(\alpha, \beta)}(x)= \\
& \frac{\Gamma(\alpha+1)}{\Gamma(\alpha+\beta+n+1)} \sum_{k=0}^{n}\left(\begin{array}{l}
n \\
k
\end{array}\right) \frac{\Gamma(\alpha+\beta+n+k+1)}{\Gamma(\alpha+k+1)}\left(\frac{x-1}{2}\right)^{k},
\end{aligned}
$$

is the normalized Jacobi polynomial of degree $n$ such that $R_{n}^{(\alpha, \beta)}(1)=1$.

G. Gasper has proved in [1] the following inequality:

$$
\begin{aligned}
& \forall n \in \mathbb{N} \backslash\{0\}, \forall x \in[-1,1], \\
& \Delta_{n}^{(\alpha, \beta)}(x) \geq \frac{\beta-\alpha}{2(n+\alpha+1)(n+\beta)}(1-x)\left(R_{n}^{(\alpha, \beta)}(x)\right)^{2},
\end{aligned}
$$

with equality only for $x= \pm 1$.

This shows that for all $n \in \mathbb{N} \backslash\{0\}$, we have $\forall x \in[-1,1], \Delta_{n}^{(\alpha, \beta)}(x) \geq 0$ if and only if $-1<\alpha \leq \beta$.

In the present paper our aim is to establish some inequalities of Turán type for Jacobi-Dunkl polynomials $\psi_{m}^{(\alpha, \beta)}$ defined on $\left[-\frac{\pi}{2}, \frac{\pi}{2}\right]$ by

$$
\begin{aligned}
& \psi_{m}^{(\alpha, \beta)}(\theta)=R_{|m|}^{(\alpha, \beta)}(\cos (2 \theta)) \\
& +i \frac{\lambda_{m}^{(\alpha, \beta)}}{4(\alpha+1)} \sin (2 \theta) R_{|m|-1}^{(\alpha+1, \beta+1)}(\cos (2 \theta)),
\end{aligned}
$$

if $m \in \mathbb{Z} \backslash\{0\}$, and $\psi_{0}^{(\alpha, \beta)}(\theta)=1$, with

$$
\lambda_{m}^{(\alpha, \beta)}:=2 \operatorname{sgn}(m) \sqrt{|m|(|m|+\alpha+\beta+1)} .
$$

We recall in the second section, some basic properties of Jacobi and Jacobi-Dunkl polynomials. We also present several explicit forms of these polynomials. Finally, we establish, in the last section, some inequalities of Turán type involving $\mathfrak{R} \psi_{m}^{(\alpha, \beta)}$, $\Im \psi_{m}^{(\alpha, \beta)}$ and $\left|\psi_{m}^{(\alpha, \beta)}\right|$ (Theorems 3.4, 3.7, 3.9). More precisely,

$$
\begin{aligned}
& \forall \theta \in\left[-\frac{\pi}{2}, \frac{\pi}{2}\right], \quad\left|\psi_{m}(\theta)\right|^{2}-\Re\left(\psi_{m-1}(\theta) \overline{\psi_{m+1}(\theta)}\right) \\
& \geq \frac{\beta-\alpha}{(|m|+\alpha+1)(|m|+\beta)}(\sin \theta)^{2}\left|\psi_{m}(\theta)\right|^{2},
\end{aligned}
$$

with equality only for $\theta \in\left\{-\frac{\pi}{2}, 0, \frac{\pi}{2}\right\}$. 
Then, we deduce that

$\forall \theta \in\left[-\frac{\pi}{2}, \frac{\pi}{2}\right],\left|\psi_{m}(\theta)\right|^{2}-\mathfrak{R}\left(\psi_{m-1}(\theta) \overline{\psi_{m+1}(\theta)}\right) \geq 0$

if and only if $-1<\alpha \leq \beta$.

In the sequel, we take $\alpha>-1, \beta>-1$ and we denote by $\rho:=\alpha+\beta+1$. We drop the exponents $(\alpha, \beta)$ when there is no confusion.

\section{Preliminaries}

In this section we recall some properties for Jacobi and Jacobi-Dunkl polynomials useful to get inequalities of Turán type which occur in the study of certain entire functions. These inequalities give an idea about the size of the gap, estimates and monotonicity properties of some special functions.

\subsection{Properties of Jacobi Polynomials}

We consider the Jacobi polynomial $\varphi_{n}$ defined on $\left[0, \frac{\pi}{2}\right]$ by

$\varphi_{n}(\theta):=\varphi_{n}^{(\alpha, \beta)}(\theta):=R_{n}^{(\alpha, \beta)}(\cos (2 \theta)), n \in \mathbb{N}, \theta \in\left[0, \frac{\pi}{2}\right]$,

where $R_{n}^{(\alpha, \beta)}$ is the normalized Jacobi polynomial such that $R_{n}^{(\alpha, \beta)}(1)=1$. According t19,22], the $\varphi_{n}$ 's satisfy the following relations:

$$
\begin{gathered}
\forall n \in \mathbb{N}, \quad \varphi_{n}(0)=1 . \\
\forall n \in \mathbb{N}, \quad \varphi_{n}\left(\frac{\pi}{2}\right)=(-1)^{n} \frac{\Gamma(\alpha+1) \Gamma(\beta+n+1)}{\Gamma(\beta+1) \Gamma(\alpha+n+1)} . \\
\forall n \in \mathbb{N}, \forall \theta \in\left[0, \frac{\pi}{2}\right], \quad \varphi_{n}^{(\beta, \alpha)}(\theta)=\frac{\varphi_{n}\left(\frac{\pi}{2}-\theta\right)}{\varphi_{n}\left(\frac{\pi}{2}\right)} .
\end{gathered}
$$

For all $n \in \mathbb{N}$, the Jacobi polynomial $\varphi_{n}$ is a solution on $] 0, \frac{\pi}{2}[$ of the following differential equation: $L y=-4 n(n+\rho) y$, where

$$
\begin{aligned}
& L y:=L_{\alpha, \beta} y:=y^{\prime \prime}+[(2 \alpha+1) \cot -(2 \beta+1) \tan ] y^{\prime}, \\
& y \in C^{2}(] 0, \frac{\pi}{2}[) .
\end{aligned}
$$

In hypergeometric form, we have

$$
\begin{gathered}
\forall n \in \mathbb{N}, \forall \theta \in\left[0, \frac{\pi}{2}[,\right. \\
\varphi_{n}(\theta)={ }_{2} F_{1}\left(-n, n+\rho ; \alpha+1 ;(\sin \theta)^{2}\right),
\end{gathered}
$$

where ${ }_{2} F_{1}(a, b ; c ; z)$ is the gaussian hypergeometric function given by

$$
{ }_{2} F_{1}(a, b ; c ; z)=\sum_{n=0}^{\infty} \frac{(a)_{n}(b)_{n}}{n !(c)_{n}} z^{n},
$$

$a, b, c, z \in \mathbb{C} ; c \notin \mathbb{Z}_{-},|z|<1$,

with

$$
(a)_{n}=\left\{\begin{array}{ccc}
1 & \text { if } & n=0, \\
a(a+1)(a+2) \ldots(a+n-1) & \text { if } & n \geq 1 .
\end{array}\right.
$$

The derivative of $\varphi_{n}$ is given by

$$
\begin{gathered}
\forall n \in \mathbb{N} \backslash\{0\}, \forall \theta \in\left[0, \frac{\pi}{2}\right], \\
\frac{d}{d \theta} \varphi_{n}^{(\alpha, \beta)}(\theta)=-\frac{n(n+\rho)}{\alpha+1} \sin (2 \theta) \varphi_{n-1}^{(\alpha+1, \beta+1)}(\theta) .
\end{gathered}
$$

In particular, $\forall n \in \mathbb{N}, \frac{d}{d \theta} \varphi_{n}(0)=0$. In the Gegenbauer case $(\beta=\alpha)$, we have $\forall n \in \mathbb{N}, \forall \theta \in\left[0, \frac{\pi}{2}\right]$,

$$
\begin{aligned}
& \varphi_{2 n}^{(\alpha, \alpha)}\left(\frac{\theta}{2}\right)=\varphi_{n}^{\left(\alpha,-\frac{1}{2}\right)}(\theta) \\
& \varphi_{2 n+1}^{(\alpha, \alpha)}\left(\frac{\theta}{2}\right)=\cos \theta \varphi_{n}^{\left(\alpha, \frac{1}{2}\right)}(\theta) .
\end{aligned}
$$

Explicit formulas of $\varphi_{n}$ are given, for all $n \in \mathbb{N}$ and $\theta \in\left[0, \frac{\pi}{2}\right]$, by

$$
\begin{aligned}
& \varphi_{n}(\theta)=\sum_{k=0}^{n}(-1)^{k} a(k, n, \alpha, \beta)(\sin \theta)^{2 k} \\
& =\sum_{k=0}^{n} b(k, n, \alpha, \beta)(\cos (2 \theta))^{k},
\end{aligned}
$$

where

$$
a(k, n, \alpha, \beta)=\left(\begin{array}{l}
n \\
k
\end{array}\right) \frac{\Gamma(\alpha+1) \Gamma(\rho+n+k)}{\Gamma(\alpha+k+1) \Gamma(\rho+n)}
$$

if $n \geq 1,0 \leq k \leq n$, and $a(0,0, \alpha, \beta)=1$, and

$$
\begin{aligned}
& b(k, n, \alpha, \beta)=\frac{a(k, n, \alpha, \beta)}{2^{k}} \\
& \times{ }_{2} F_{1}\left(k-n, n+k+\rho ; \alpha+k+1 ; \frac{1}{2}\right), \quad 0 \leq k \leq n .
\end{aligned}
$$

The orthogonality property is given by

$$
\forall m, n \in \mathbb{N}, \int_{0}^{\frac{\pi}{2}} \varphi_{m}(\theta) \varphi_{n}(\theta) A(\theta) d \theta=k_{n}^{-1} \delta_{m n},
$$

where

$$
k_{n}:=k_{n}^{(\alpha, \beta)}:=\frac{(2 n+\rho) \Gamma(\alpha+n+1) \Gamma(\rho+n)}{2^{2 \rho-1}(\Gamma(\alpha+1))^{2} n ! \Gamma(\beta+n+1)},
$$

if $n \in \mathbb{N} \backslash\{0\}$, and 


$$
k_{0}:=k_{0}^{(\alpha, \beta)}:=\frac{\Gamma(\rho+1)}{2^{2 \rho-1} \Gamma(\alpha+1) \Gamma(\beta+1)} .
$$

We also have, for all $n \in \mathbb{N} \backslash\{0\}$ and $\theta \in\left[0, \frac{\pi}{2}\right]$, the following recurrence formula:

$$
\begin{aligned}
& \varphi_{n+1}(\theta)= \\
& \frac{(2 n+\rho)\left[(2 n+\rho+1)(2 n+\rho-1) \cos (2 \theta)+\alpha^{2}-\beta^{2}\right]}{2(n+\alpha+1)(n+\rho)(2 n+\rho-1)} \varphi_{n}(\theta) \\
& -\frac{n(n+\beta)(2 n+\rho+1)}{(n+\alpha+1)(n+\rho)(2 n+\rho-1)} \varphi_{n-1}(\theta) .
\end{aligned}
$$

Examples 2.1 For all $n \in \mathbb{N}$ and $\theta \in\left[0, \frac{\pi}{2}\right]$, we have

1) $\varphi_{0}(\theta)=1, \varphi_{1}(\theta)=1-\frac{\rho+1}{\alpha+1}(\sin \theta)^{2}$,

$$
\varphi_{2}(\theta)=\frac{(\rho+2)(\rho+3)}{(\alpha+1)(\alpha+2)}(\sin \theta)^{4}-\frac{2(\rho+2)}{\alpha+1}(\sin \theta)^{2}+1 .
$$

2) $\varphi_{n}^{\left(-\frac{1}{2},-\frac{1}{2}\right)}(\theta)=\cos (2 n \theta)$,

$$
\varphi_{n}^{\left(\frac{1}{2},-\frac{1}{2}\right)}(\theta)=\frac{\sin ((2 n+1) \theta)}{(2 n+1) \sin \theta}, \theta \neq 0 .
$$

3) $\varphi_{n}^{\left(-\frac{1}{2}, \frac{1}{2}\right)}(\theta)=\frac{\cos ((2 n+1) \theta)}{\cos \theta}, \theta \neq \frac{\pi}{2}$,

$$
\varphi_{n}^{\left(\frac{1}{2}, \frac{1}{2}\right)}(\theta)=\frac{\sin (2(n+1) \theta)}{(n+1) \sin (2 \theta)}, \theta \neq 0, \frac{\pi}{2} .
$$

\subsection{Properties of Jacobi-Dunkl Polynomials}

We take $\varphi_{n}(\theta):=\varphi_{n}(-\theta)$ for all $n \in \mathbb{N}$ and $\theta \in\left[-\frac{\pi}{2}, 0[, \quad A(0)=0\right.$, and $A(\theta):=A(-\theta)$ for all $\theta \in]-\frac{\pi}{2}, 0[$.

For all $m \in \mathbb{Z}$, the Jacobi-Dunkl polynomial $\psi_{m}:=\psi_{m}^{(\alpha, \beta)}$ is defined on $\left[-\frac{\pi}{2}, \frac{\pi}{2}\right]$ by $\forall \theta \in\left[-\frac{\pi}{2}, \frac{\pi}{2}\right]$,

$$
\psi_{m}(\theta):=\varphi_{|m|}(\theta)+i \frac{\lambda_{m}}{4(\alpha+1)} \sin (2 \theta) \varphi_{|m|-1}^{(\alpha+1, \beta+1)}(\theta),
$$

if $m \in \mathbb{Z} \backslash\{0\}$, and $\psi_{0}^{(\alpha, \beta)}(\theta)=1$,

with $\lambda_{m}:=\lambda_{m}^{(\alpha, \beta)}:=2 \operatorname{sgn}(m) \sqrt{|m|(|m|+\rho)}$ (see [22]).

\section{Remarks 2.2}

$$
\text { 1) } \begin{array}{rl}
\forall m & \mathbb{Z} \backslash\{0\}, \forall \theta \in\left[-\frac{\pi}{2}, \frac{\pi}{2}\right], \\
& \psi_{m}(\theta)=\varphi_{|m|}(\theta)-\frac{i}{\lambda_{m}} \frac{d}{d \theta} \varphi_{|m|}(\theta) .
\end{array}
$$

2) In [22], the author has studied the harmonic analysis associated with these polynomials.

Proposition 2.3 For all $m \in \mathbb{Z}$ and $\theta \in\left[-\frac{\pi}{2}, \frac{\pi}{2}\right]$, we have

1) $\psi_{m}(0)=1, \quad \psi_{-m}(-\theta)=\psi_{m}(\theta)$,

$$
\overline{\psi_{m}(\theta)}=\psi_{-m}(\theta)=\psi_{m}(-\theta) \text {. }
$$

2) $\mathfrak{R} \psi_{m}(\theta)=\frac{\psi_{m}(\theta)+\psi_{m}(-\theta)}{2}=\varphi_{|m|}(\theta)$.

3)

$$
\begin{aligned}
& \Im \psi_{m}(\theta)=\frac{\psi_{m}(\theta)-\psi_{m}(-\theta)}{2} \\
& =\frac{\lambda_{m}}{4(\alpha+1)} \sin (2 \theta) \varphi_{|m|-1}^{(\alpha+1, \beta+1)}(\theta), m \neq 0
\end{aligned}
$$

4)

$$
\psi_{2 m}^{(\alpha, \alpha)}\left(\frac{\theta}{2}\right)=\psi_{m}^{\left(\alpha,-\frac{1}{2}\right)}(\theta) .
$$

5)

$$
\begin{aligned}
& \psi_{m}\left(\frac{\pi}{2}\right)=\psi_{m}\left(-\frac{\pi}{2}\right)=\varphi_{|m|}\left(\frac{\pi}{2}\right) \\
& =(-1)^{m} \frac{\Gamma(\alpha+1) \Gamma(\beta+|m|+1)}{\Gamma(\beta+1) \Gamma(\alpha+|m|+1)}
\end{aligned}
$$

6)

$$
\begin{gathered}
\psi_{m}^{(\beta, \alpha)}(\theta)=\frac{\psi_{m}\left(\theta-\frac{\pi}{2}\right)}{\psi_{m}\left(\frac{\pi}{2}\right)}, 0 \leq \theta \leq \frac{\pi}{2}, \\
\psi_{m}^{(\beta, \alpha)}(\theta)=\frac{\psi_{m}\left(\theta+\frac{\pi}{2}\right)}{\psi_{m}\left(\frac{\pi}{2}\right)},-\frac{\pi}{2} \leq \theta<0 .
\end{gathered}
$$

Proof: We use (1), (9), (4), (2) and (3) to get the above formulas.

For all $m \in \mathbb{Z}, \psi_{m}$ is the unique $C^{\infty}$ solution on ]$-\frac{\pi}{2}, \frac{\pi}{2}[$ of the differential-difference equation

$$
\left\{\begin{array}{ccc}
\Lambda u(\theta) & = & i \lambda_{m} u(\theta) \\
u(0) & = & 1
\end{array}\right.
$$

where $\Lambda:=\Lambda_{\alpha, \beta}$ is the Jacobi-Dunkl operator defined on $C^{1}(]-\frac{\pi}{2}, \frac{\pi}{2}[)$ by

$$
\begin{aligned}
\Lambda u(\theta):= & u^{\prime}(\theta)+[(2 \alpha+1) \cot \theta-(2 \beta+1) \tan \theta] \\
& \times \frac{u(\theta)-u(-\theta)}{2}, \quad u \in C^{1}(]-\frac{\pi}{2}, \frac{\pi}{2}[) .
\end{aligned}
$$

In the following proposition we give explicit forms of $\psi_{m}$. 
Proposition 2.4 For all $m \in \mathbb{Z} \backslash\{0\}$ and $\theta \in\left[-\frac{\pi}{2}, \frac{\pi}{2}\right]$, we have

1)

$$
\begin{aligned}
& \psi_{m}(\theta)=\sum_{k=0}^{|m|}(-1)^{k} a(k,|m|, \alpha, \beta) \\
& \times\left[1+i \frac{(|m|-k)(|m|+k+\rho)}{\lambda_{m}(k+\alpha+1)} \sin (2 \theta)\right](\sin \theta)^{2 k}
\end{aligned}
$$

2)

$$
\begin{aligned}
\psi_{m}(\theta) & =\frac{\Gamma(\alpha+1) \Gamma(\rho+2|m|)}{2^{|m|} \Gamma(\alpha+|m|+1) \Gamma(\rho+|m|)}(\cos (2 \theta))^{|m|} \\
& +\sum_{k=0}^{|m|-1}[b(k,|m|, \alpha, \beta) \\
& \left.+i \frac{2(k+1) b(k+1,|m|, \alpha, \beta)}{\lambda_{m}} \sin (2 \theta)\right](\cos (2 \theta))^{k},
\end{aligned}
$$

where $a(k, n, \alpha, \beta)$ and $b(k, n, \alpha, \beta), n \in \mathbb{N}, 0 \leq k \leq n$, are respectively given by (6) and (7).

Proof: We obtain the above formulas from (9) and (5).

The orthogonality property is given in [22] by $\forall m, n \in \mathbb{Z}, \int_{-\frac{\pi}{2}}^{\frac{\pi}{2}} \psi_{m}(\theta) \overline{\psi_{n}(\theta)} A(\theta) d \theta=h_{m}^{-1} \delta_{m n}$, with

$$
h_{m}:=h_{m}^{(\alpha, \beta)}:=\left(\int_{-\frac{\pi}{2}}^{\frac{\pi}{2}}\left|\psi_{m}(\theta)\right|^{2} A(\theta) d \theta\right)^{-1}=\frac{k_{|m|}}{4},
$$

$m \in \mathbb{Z} \backslash\{0\}$ and $h_{0}:=h_{0}^{(\alpha, \beta)}:=\frac{k_{0}}{2}$, where $k_{n}, n \in \mathbb{N}$, is given by (8).

Examples 2.5 For all $m \in \mathbb{Z}$ and $\theta \in\left[-\frac{\pi}{2}, \frac{\pi}{2}\right]$, we have

1) $\psi_{0}(\theta)=1$,

$$
\psi_{1}(\theta)=1-\frac{\rho+1}{\alpha+1}(\sin \theta)^{2}+i \frac{\sqrt{\rho+1}}{2(\alpha+1)} \sin (2 \theta) .
$$

2)

$$
\begin{aligned}
& \psi_{2}(\theta)=\frac{(\rho+2)(\rho+3)}{(\alpha+1)(\alpha+2)}(\sin \theta)^{4}-\frac{2(\rho+2)}{\alpha+1}(\sin \theta)^{2}+1 \\
& +i \frac{\sqrt{2(\rho+2)}}{2(\alpha+1)(\alpha+2)} \sin (2 \theta)\left[\alpha+2-(\rho+3)(\sin \theta)^{2}\right] . \\
& \text { 3) } \psi_{m}^{\left(-\frac{1}{2},-\frac{1}{2}\right)}(\theta)=e^{2 i m \theta} .
\end{aligned}
$$

4)

$$
\begin{aligned}
& \psi_{m}^{\left(\frac{1}{2},-\frac{1}{2}\right)}(\theta)=\frac{\sin ((2|m|+1) \theta)}{(2|m|+1) \sin \theta} \\
& +\frac{i}{2(2|m|+1) \sqrt{|m|(|m|+1)}} \\
& \times\left[\left(2|m|+1+(\cot \theta)^{2}\right) \sin (2 m \theta)-2 m \cot \theta \cos (2 m \theta)\right], \\
& m \neq 0, \theta \neq 0
\end{aligned}
$$

5)

$$
\begin{aligned}
& \psi_{m}^{\left(-\frac{1}{2}, \frac{1}{2}\right)}(\theta)=\frac{\cos ((2|m|+1) \theta)}{\cos \theta}+\frac{i}{2 \sqrt{|m|(|m|+1)}} \\
& \times\left[\left(2|m|+1+(\tan \theta)^{2}\right) \sin (2 m \theta)+2 m \tan \theta \cos (2 m \theta)\right], \\
& m \neq 0, \theta \neq \pm \frac{\pi}{2} .
\end{aligned}
$$

$$
\begin{aligned}
& \psi_{m}^{\left(\frac{1}{2}, \frac{1}{2}\right)}(\theta)=\frac{\sin (2(|m|+1) \theta)}{(|m|+1) \sin (2 \theta)}+\frac{i}{(|m|+1) \sqrt{|m|(|m|+2)}} \\
& \times\left[\left(|m|+1+(\cot (2 \theta))^{2}\right) \sin (2 m \theta)-m \cot (2 \theta) \cos (2 m \theta)\right], \\
& m \neq 0, \theta \neq 0, \pm \frac{\pi}{2} .
\end{aligned}
$$

\section{Turán Type Inequalities}

\subsection{General Case}

G. Gasper has proved in [1] the following proposition: Proposition 3.1 For all $n \in \mathbb{N} \backslash\{0\}$, we have

1)

$$
\begin{aligned}
& \forall \theta \in\left[0, \frac{\pi}{2}\right],\left(\varphi_{n}(\theta)\right)^{2}-\varphi_{n-1}(\theta) \varphi_{n+1}(\theta) \\
& \geq \frac{\beta-\alpha}{(n+\alpha+1)(n+\beta)}(\sin \theta)^{2}\left(\varphi_{n}(\theta)\right)^{2},
\end{aligned}
$$

with equality only for $\theta \in\left\{0, \frac{\pi}{2}\right\}$.

2) $\forall \theta \in\left[0, \frac{\pi}{2}\right], \quad\left(\varphi_{n}(\theta)\right)^{2}-\varphi_{n-1}(\theta) \varphi_{n+1}(\theta) \geq 0$ if and only if $-1<\alpha \leq \beta$.

In the sequel if this subsection, let $m$ be an integer not belonging to $\{-1,0,1\}$.

\section{Lemma 3.2}

$$
\begin{aligned}
& \text { 1) } \forall p \in\{-1,1\}, \frac{\lambda_{p-1} \lambda_{p+1}}{\lambda_{p}^{2}}=0 . \\
& \text { 2) } 0<\frac{\lambda_{m-1} \lambda_{m+1}}{\lambda_{m}^{2}}<1 .
\end{aligned}
$$

Proof: Note that $\lambda_{m-1} \lambda_{m+1}=\lambda_{|m|-1} \lambda_{|m|+1}>0$ and

$$
\frac{\lambda_{m}^{4}-\lambda_{m-1}^{2} \lambda_{m+1}^{2}}{16}=2 m^{2}+2 \rho|m|+\rho^{2}-1 .
$$

If $2-\rho^{2}<0$, then $2 m^{2}+2 \rho|m|+\rho^{2}-1>0$.

If $2-\rho^{2}=0$, then $-\frac{\rho}{2}<1<|m|$ and

$$
2 m^{2}+2 \rho|m|+\rho^{2}-1>0
$$

If $2-\rho^{2}>0$, then 


$$
\frac{-\rho-\sqrt{2-\rho^{2}}}{2}<\frac{-\rho+\sqrt{2-\rho^{2}}}{2}<1<|m|
$$

and $2 m^{2}+2 \rho|m|+\rho^{2}-1>0$.

\section{Lemma 3.3}

$$
\begin{aligned}
& \forall n \in \mathbb{N} \backslash\{0\}, \forall \theta \in\left[-\frac{\pi}{2}, \frac{\pi}{2}\right], \\
& \frac{\beta-\alpha}{(n+\alpha+1)(n+\beta)}(\sin \theta)^{2}<1 .
\end{aligned}
$$

Proof: Let $n \in \mathbb{N} \backslash\{0\}$ and $\theta \in\left[-\frac{\pi}{2}, \frac{\pi}{2}\right]$.

$$
\begin{aligned}
& (n+\alpha+1)(n+\beta)-(\beta-\alpha)(\sin \theta)^{2} \\
& =n^{2}+\rho n+(\alpha-\beta)(\sin \theta)^{2}+(\alpha+1) \beta .
\end{aligned}
$$

If $\Delta:=\rho^{2}-4\left[(\alpha-\beta)(\sin \theta)^{2}+(\alpha+1) \beta\right]<0 \quad, \quad$ then $n^{2}+\rho n+(\alpha-\beta)(\sin \theta)^{2}+(\alpha+1) \beta>0$.

If $\Delta=0$, then $-\frac{\rho}{2}<1<n$ and

$$
n^{2}+\rho n+(\alpha-\beta)(\sin \theta)^{2}+(\alpha+1) \beta>0 \text {. }
$$

If $\Delta>0$, then $\frac{-\rho-\sqrt{\Delta}}{2}<\frac{-\rho+\sqrt{\Delta}}{2}$ and

$$
\frac{(\rho+2)^{2}-\Delta}{4}
$$$$
=(\alpha+1)(\sin \theta)^{2}+(\beta+1)(\cos \theta)^{2}+(\alpha+1)(\beta+1)>0 .
$$

Hence $\frac{-\rho+\sqrt{\Delta}}{2}<1<n$ and

$$
n^{2}+\rho n+(\alpha-\beta)(\sin \theta)^{2}+(\alpha+1) \beta>0 .
$$

The following theorem gives a Turán type inequality for $\mathfrak{I} \psi_{m}$.

\section{Theorem 3.4}

$$
\begin{aligned}
& \forall \theta \in\left[-\frac{\pi}{2}, \frac{\pi}{2}\right],\left(\mathfrak{I} \psi_{m}(\theta)\right)^{2}-\mathfrak{J} \psi_{m-1}(\theta) \mathfrak{I} \psi_{m+1}(\theta) \\
& \geq\left[\begin{array}{l}
\frac{\lambda_{m-1} \lambda_{m+1}}{\lambda_{m}^{2}} \frac{\beta-\alpha}{(|m|+\alpha+1)(|m|+\beta)}(\sin \theta)^{2} \\
+1-\frac{\lambda_{m-1} \lambda_{m+1}}{\lambda_{m}^{2}}
\end{array}\right] \times\left(\mathfrak{I} \psi_{m}(\theta)\right)^{2},
\end{aligned}
$$

with equality only for $\theta \in\left\{-\frac{\pi}{2}, 0, \frac{\pi}{2}\right\}$.

Proof: Let $\theta \in\left[-\frac{\pi}{2}, \frac{\pi}{2}\right]$.

$\left(\mathfrak{I} \psi_{m}(\theta)\right)^{2}-\mathfrak{J} \psi_{m-1}(\theta) \mathfrak{I} \psi_{m+1}(\theta)$

$=\left(\frac{\sin (2 \theta)}{4(\alpha+1)}\right)^{2}\left(\begin{array}{l}\lambda_{m \mid}^{2}\left(\varphi_{|m|-1}^{(\alpha+1, \beta+1)}(\theta)\right)^{2} \\ -\lambda_{|m|-1} \lambda_{|m|+1} \varphi_{|m|-2}^{(\alpha+1, \beta+1)}(\theta) \varphi_{|m|}^{(\alpha+1, \beta+1)}(\theta)\end{array}\right)$

$$
\begin{aligned}
& =\left(\frac{\lambda_{m} \sin (2 \theta)}{4(\alpha+1)}\right)^{2}\left[\left(1-\frac{\lambda_{|m|-1} \lambda_{|m|+1}}{\lambda_{|m|}^{2}}\right)\left(\varphi_{|m|-1}^{(\alpha+1, \beta+1)}(\theta)\right)^{2}\right. \\
& \left.+\frac{\lambda_{m \mid-1} \lambda_{m \mid+1}}{\lambda_{|m|}^{2}}\left(\begin{array}{l}
\left.\varphi_{|m|-1}^{(\alpha+1, \beta+1)}(\theta)\right)^{2} \\
-\varphi_{|m|-2}^{(\alpha+1, \beta+1)}(\theta) \varphi_{|m|}^{(\alpha+1, \beta+1)}(\theta)
\end{array}\right)\right] .
\end{aligned}
$$

Proposition 3.1 and Lemma 3.2 give the above inequality.

\section{Corollary $\mathbf{3 . 5}$}

$$
\begin{gathered}
\forall \theta \in\left[-\frac{\pi}{2}, \frac{\pi}{2}\right], \\
\mathfrak{I} \psi_{m-1}(\theta) \mathfrak{I} \psi_{m+1}(\theta) \leq \frac{\lambda_{m-1} \lambda_{m+1}}{\lambda_{m}^{2}}\left(\mathfrak{I} \psi_{m}(\theta)\right)^{2}
\end{gathered}
$$

if and only if $-1<\alpha \leq \beta$.

\section{Remark 3.6}

$$
\forall \theta \in\left[-\frac{\pi}{2}, \frac{\pi}{2}\right]
$$

$\left(\frac{\lambda_{m} \sin (2 \theta)}{4(\alpha+1)}\right)^{2} \min \left\{\varphi_{|m|-2}^{(\alpha+1, \beta+1)}(\theta) \varphi_{|m|}^{(\alpha+1, \beta+1)}(\theta), 0\right\}$ $\leq \mathfrak{I} \psi_{m-1}(\theta) \mathfrak{I} \psi_{m+1}(\theta)$

$=\left(\frac{\lambda_{m} \sin (2 \theta)}{4(\alpha+1)}\right)^{2} \frac{\lambda_{|m|-1} \lambda_{|m|+1}}{\lambda_{|m|}^{2}} \varphi_{|m|-2}^{(\alpha+1, \beta+1)}(\theta) \varphi_{|m|}^{(\alpha+1, \beta+1)}(\theta)$ $\leq\left(\frac{\lambda_{m} \sin (2 \theta)}{4(\alpha+1)}\right)^{2} \max \left\{\varphi_{|m|-2}^{(\alpha+1, \beta+1)}(\theta) \varphi_{|m|}^{(\alpha+1, \beta+1)}(\theta), 0\right\}$.

We establish now the following Turán type inequality for the Jacobi-Dunkl polynomials $\psi_{m}$.

Theorem 3.7

$$
\begin{aligned}
& \forall \theta \in\left[-\frac{\pi}{2}, \frac{\pi}{2}\right], \\
& \left|\psi_{m}(\theta)\right|^{2}-\mathfrak{R}\left(\psi_{m-1}(\theta) \overline{\psi_{m+1}(\theta)}\right) \\
& \geq \frac{\beta-\alpha}{(|m|+\alpha+1)(|m|+\beta)}(\sin \theta)^{2}\left|\psi_{m}(\theta)\right|^{2},
\end{aligned}
$$

with equality only for $\theta \in\left\{-\frac{\pi}{2}, 0, \frac{\pi}{2}\right\}$.

Proof: Let $\theta \in\left[-\frac{\pi}{2}, \frac{\pi}{2}\right]$.

$$
\left|\psi_{m}(\theta)\right|^{2}-\mathfrak{R}\left(\psi_{m-1}(\theta) \overline{\psi_{m+1}(\theta)}\right)
$$

$=\left(\varphi_{|m|}(\theta)\right)^{2}+\left(\mathfrak{I} \psi_{m}(\theta)\right)^{2}-\varphi_{|m-1|}(\theta) \varphi_{|m+1|}(\theta)$

$-\lambda_{m-1} \lambda_{m+1}\left(\frac{\sin (2 \theta)}{4(\alpha+1)}\right)^{2} \varphi_{|m-1|-1}^{(\alpha+1, \beta+1)}(\theta) \varphi_{|m+1|-1}^{(\alpha+1, \beta+1)}(\theta)$

$=\frac{\beta-\alpha}{(|m|+\alpha+1)(|m|+\beta)}(\sin \theta)^{2}\left|\psi_{m}(\theta)\right|^{2}$

$+\left(\varphi_{|m|}(\theta)\right)^{2}-\varphi_{|m-1|}(\theta) \varphi_{|m+1|}(\theta)$ 


$$
\begin{aligned}
& -\frac{\beta-\alpha}{(|m|+\alpha+1)(|m|+\beta)}(\sin \theta)^{2}\left(\varphi_{|m|}(\theta)\right)^{2} \\
& +\lambda_{|m|-1} \lambda_{|m|+1}\left(\frac{\sin (2 \theta)}{4(\alpha+1)}\right)^{2} \\
& \times\left[\left(\varphi_{|m|-1}^{(\alpha+1, \beta+1)}(\theta)\right)^{2}-\varphi_{|m|-2}^{(\alpha+1, \beta+1)}(\theta) \varphi_{|m|}^{(\alpha+1, \beta+1)}(\theta)\right. \\
& -\frac{(\beta+1)-(\alpha+1)}{((|m|-1)+(\alpha+1)+1)((|m|-1)+(\beta+1))} \\
& \left.\times(\sin \theta)^{2}\left(\varphi_{|m|-1}^{(\alpha+1, \beta+1)}(\theta)\right)^{2}\right] \\
& +\left(1-\frac{\left.\lambda_{m \mid-1} \lambda_{|m|+1}\right)}{\lambda_{|m|}^{2}}\right) \\
& \times\left(1-\frac{\beta-\alpha}{(|m|+\alpha+1)(|m|+\beta)}(\sin \theta)^{2}\right)\left(\mathfrak{J} \psi_{m}(\theta)\right)^{2} .
\end{aligned}
$$

From Proposition 3.1 it is obvious that

$$
\begin{aligned}
& \left(\varphi_{|m|}(\theta)\right)^{2}-\varphi_{|m-1|}(\theta) \varphi_{|m+1|}(\theta) \\
& -\frac{\beta-\alpha}{(|m|+\alpha+1)(|m|+\beta)}(\sin \theta)^{2}\left(\varphi_{|m|}(\theta)\right)^{2} \geq 0,
\end{aligned}
$$

and

$$
\begin{aligned}
& \left(\varphi_{|m|-1}^{(\alpha+1, \beta+1)}(\theta)\right)^{2}-\varphi_{|m|-2}^{(\alpha+1, \beta+1)}(\theta) \varphi_{|m|}^{(\alpha+1, \beta+1)}(\theta) \\
& -\frac{(\beta+1)-(\alpha+1)}{((|m|-1)+(\alpha+1)+1)((|m|-1)+(\beta+1))} \\
& \times(\sin \theta)^{2}\left(\varphi_{|m|-1}^{(\alpha+1, \beta+1)}(\theta)\right)^{2} \geq 0 .
\end{aligned}
$$

Note that $\lambda_{m-1} \lambda_{m+1}=\lambda_{|m|-1} \lambda_{|m|+1}>0$ and, by Lemma 3.2, $0<1-\frac{\lambda_{m \mid-1} \lambda_{|m|+1}}{\lambda_{|m|}^{2}}<1$.

To finish the proof we use Lemma 3.3.

\section{Corollary 3.8}

$$
\forall \theta \in\left[-\frac{\pi}{2}, \frac{\pi}{2}\right],\left|\psi_{m}(\theta)\right|^{2}-\mathfrak{R}\left(\psi_{m-1}(\theta) \overline{\psi_{m+1}(\theta)}\right) \geq 0
$$

if and only if $-1<\alpha \leq \beta$.

Another inequality of Turán type is given in the following theorem:

\section{Theorem 3.9}

$$
\begin{aligned}
& \forall \theta \in\left[-\frac{\pi}{2}, \frac{\pi}{2}\right], \\
& \left(\mathfrak{R} \psi_{m}(\theta)\right)^{2}+\frac{\lambda_{m-1} \lambda_{m+1}}{\lambda_{m}^{2}}\left(\mathfrak{I} \psi_{m}(\theta)\right)^{2} \\
& \geq \frac{\beta-\alpha}{(|m|+\alpha+1)(|m|+\beta)}(\sin \theta)^{2} \\
& \times\left[\left(\mathfrak{R} \psi_{m}(\theta)\right)^{2}+\frac{\lambda_{m-1} \lambda_{m+1}}{\lambda_{m}^{2}}\left(\mathfrak{I} \psi_{m}(\theta)\right)^{2}\right],
\end{aligned}
$$

with equality only for $\theta \in\left\{-\frac{\pi}{2}, 0, \frac{\pi}{2}\right\}$.

$$
\begin{aligned}
& \text { Proof: Let } \theta \in\left[-\frac{\pi}{2}, \frac{\pi}{2}\right] \\
& \left(\mathfrak{R} \psi_{m}(\theta)\right)^{2}+\frac{\lambda_{m-1} \lambda_{m+1}}{\lambda_{m}^{2}}\left(\mathfrak{I} \psi_{m}(\theta)\right)^{2} \\
& -\mathfrak{R}\left(\psi_{m-1}(\theta) \overline{\psi_{m+1}(\theta)}\right) \\
& =\frac{\beta-\alpha}{(|m|+\alpha+1)(|m|+\beta)}(\sin \theta)^{2} \\
& \times\left[\left(\mathfrak{R} \psi_{m}(\theta)\right)^{2}+\frac{\lambda_{m-1} \lambda_{m+1}}{\lambda_{m}^{2}}\left(\mathfrak{I} \psi_{m}(\theta)\right)^{2}\right] \\
& +\left(\varphi_{|m|}(\theta)\right)^{2}-\varphi_{|m-1|}(\theta) \varphi_{|m+1|}(\theta) \\
& -\frac{\beta-\alpha}{(|m|+\alpha+1)(|m|+\beta)}(\sin \theta)^{2}\left(\varphi_{|m|}(\theta)\right)^{2} \\
& +\lambda_{|m|-1} \lambda_{|m|+1}\left(\frac{\sin (2 \theta)}{4(\alpha+1)}\right)^{2} \\
& \times\left[\left(\varphi_{|m|-1}^{(\alpha+1, \beta+1)}(\theta)\right)^{2}-\varphi_{|m|-2}^{(\alpha+1, \beta+1)}(\theta) \varphi_{|m|}^{(\alpha+1, \beta+1)}(\theta)\right. \\
& -\frac{(\beta+1)-(\alpha+1)}{((|m|-1)+(\alpha+1)+1)((|m|-1)+(\beta+1))} \\
& \left.\times(\sin \theta)^{2}\left(\varphi_{|m|-1}^{(\alpha+1, \beta+1)}(\theta)\right)^{2}\right] .
\end{aligned}
$$

Then we complete the proof as in that of Theorem 3.7.

\section{Corollary 3.10}

$$
\begin{aligned}
& \forall \theta \in\left[-\frac{\pi}{2}, \frac{\pi}{2}\right], \\
& \mathfrak{R}\left(\psi_{m-1}(\theta) \overline{\psi_{m+1}(\theta)}\right) \\
& \leq\left(\mathfrak{R} \psi_{m}(\theta)\right)^{2}+\frac{\lambda_{m-1} \lambda_{m+1}}{\lambda_{m}^{2}}\left(\mathfrak{I} \psi_{m}(\theta)\right)^{2},
\end{aligned}
$$

if and only if $-1<\alpha \leq \beta$.

Bu using (3) and (2), Proposition 3.1 may be rewritten in the following form:

Proposition 3.11 For all $n \in \mathbb{N} \backslash\{0\}$, we have

$$
\text { 1) } \begin{aligned}
& \forall \forall \in\left[-\frac{\pi}{2}, \frac{\pi}{2}\right], \\
& \frac{(n+\alpha)(n+\beta+1)}{(n+\alpha+1)(n+\beta)}\left(\varphi_{n}(\theta)\right)^{2}-\varphi_{n-1}(\theta) \varphi_{n+1}(\theta) \\
& \geq \frac{\alpha-\beta}{(n+\alpha+1)(n+\beta)}(\cos \theta)^{2}\left(\varphi_{n}(\theta)\right)^{2},
\end{aligned}
$$

with equality only for $\theta \in\left\{0, \frac{\pi}{2}\right\}$.

$$
\text { 2) } \begin{aligned}
\forall \theta \in\left[0, \frac{\pi}{2}\right], \\
\varphi_{n-1}(\theta) \varphi_{n+1}(\theta) \leq \frac{(n+\alpha)(n+\beta+1)}{(n+\alpha+1)(n+\beta)}\left(\varphi_{n}(\theta)\right)^{2}
\end{aligned}
$$

if and only if $-1<\beta \leq \alpha$.

From (12) and (11), we deduce the following proposition which gives again Turán type inequalities for $\mathfrak{J} \psi_{m}$. 


\section{Proposition 3.12}

1) $\forall \theta \in\left[-\frac{\pi}{2}, \frac{\pi}{2}\right]$,

$\frac{(|m|+\alpha)(|m|+\beta+1)}{(|m|+\alpha+1)(|m|+\beta)}\left(\mathfrak{I} \psi_{m}(\theta)\right)^{2}-\mathfrak{I} \psi_{m-1}(\theta) \mathfrak{I} \psi_{m+1}(\theta)$

$\geq\left[\begin{array}{l}\frac{\lambda_{m-1} \lambda_{m+1}}{\lambda_{m}^{2}} \frac{\alpha-\beta}{(|m|+\alpha)(|m|+\beta+1)}(\cos \theta)^{2} \\ +1-\frac{\lambda_{m-1} \lambda_{m+1}}{\lambda_{m}^{2}}\end{array}\right]$

$\times \frac{(|m|+\alpha)(|m|+\beta+1)}{(|m|+\alpha+1)(|m|+\beta)}\left(\Im \psi_{m}(\theta)\right)^{2}$,

with equality only for $\theta \in\left\{-\frac{\pi}{2}, 0, \frac{\pi}{2}\right\}$.

2) $\forall \theta \in\left[-\frac{\pi}{2}, \frac{\pi}{2}\right]$,

$$
\begin{aligned}
& \\
& \Im \psi_{m-1}(\theta) \mathfrak{I} \psi_{m+1}(\theta) \\
& \leq \frac{\lambda_{m-1} \lambda_{m+1}}{\lambda_{m}^{2}} \frac{(|m|+\alpha)(|m|+\beta+1)}{(|m|+\alpha+1)(|m|+\beta)}\left(\mathfrak{I} \psi_{m}(\theta)\right)^{2}
\end{aligned}
$$

if and only if $-1<\beta \leq \alpha$.

It is obvious that Theorem 3.7 and Corollary 3.8 may be rewritten in the following proposition:

\section{Proposition 3.13}

$$
\begin{aligned}
& \text { 1) } \forall \theta \in\left[-\frac{\pi}{2}, \frac{\pi}{2}\right], \\
& \frac{(|m|+\alpha)(|m|+\beta+1)}{(|m|+\alpha+1)(|m|+\beta)}\left|\psi_{m}(\theta)\right|^{2}-\Re\left(\psi_{m-1}(\theta) \overline{\psi_{m+1}(\theta)}\right) \\
& \geq \frac{\alpha-\beta}{(|m|+\alpha+1)(|m|+\beta)}(\cos \theta)^{2}\left|\psi_{m}(\theta)\right|^{2},
\end{aligned}
$$

with equality only for $\theta \in\left\{-\frac{\pi}{2}, 0, \frac{\pi}{2}\right\}$.

$$
\text { 2) } \forall \theta \in\left[-\frac{\pi}{2}, \frac{\pi}{2}\right] \text {, }
$$

$\mathfrak{R}\left(\psi_{m-1}(\theta) \overline{\psi_{m+1}(\theta)}\right) \leq \frac{(|m|+\alpha)(|m|+\beta+1)}{(|m|+\alpha+1)(|m|+\beta)}\left|\psi_{m}(\theta)\right|^{2}$.

if and only if $-1<\beta \leq \alpha$.

Also, Theorem 3.9 and Corollary 3.10 may be rewritten in the following form:

\section{Proposition 3.14}

$$
\begin{aligned}
& \text { 1) } \forall \theta \in\left[-\frac{\pi}{2}, \frac{\pi}{2}\right], \\
& \frac{(|m|+\alpha)(|m|+\beta+1)}{(|m|+\alpha+1)(|m|+\beta)}\left[\begin{array}{l}
\left(\Re \psi_{m}(\theta)\right)^{2} \\
+\frac{\lambda_{m-1} \lambda_{m+1}}{\lambda_{m}^{2}}\left(\Im \psi_{m}(\theta)\right)^{2}
\end{array}\right] \\
& -\Re\left(\psi_{m-1}(\theta) \overline{\psi_{m+1}(\theta)}\right) \\
& \geq \frac{\alpha-\beta}{(|m|+\alpha+1)(|m|+\beta)}(\cos \theta)^{2}
\end{aligned}
$$

$$
\times\left[\left(\Re \psi_{m}(\theta)\right)^{2}+\frac{\lambda_{m-1} \lambda_{m+1}}{\lambda_{m}^{2}}\left(\Im \psi_{m}(\theta)\right)^{2}\right]
$$

with equality only for $\theta \in\left\{-\frac{\pi}{2}, 0, \frac{\pi}{2}\right\}$.

$$
\begin{aligned}
& \text { 2) } \forall \theta \in\left[-\frac{\pi}{2}, \frac{\pi}{2}\right], \\
& \Re\left(\psi_{m-1}(\theta) \overline{\psi_{m+1}(\theta)}\right) \\
& \leq \frac{(|m|+\alpha)(|m|+\beta+1)}{(|m|+\alpha+1)(|m|+\beta)}\left[\begin{array}{l}
\left(\Re \psi_{m}(\theta)\right)^{2} \\
+\frac{\lambda_{m-1} \lambda_{m+1}}{\lambda_{m}^{2}}\left(\Im \psi_{m}(\theta)\right)^{2}
\end{array}\right]
\end{aligned}
$$

if and only if $-1<\beta \leq \alpha$.

\subsection{Special Cases}

From (4), Proposition 3.1, and Proposition 3.11, it is clear that the Gegenbauer polynomials $\varphi_{n}^{(\alpha, \alpha)}, n \in \mathbb{N}$, satisfy the following inequalities:

Proposition 3.15 For all $n \in \mathbb{N} \backslash\{0\}$, we have

$$
\text { 1) } \begin{aligned}
\forall \theta & \in\left[0, \frac{\pi}{4}\right], \\
& \left(\varphi_{2 n}^{(\alpha, \alpha)}(\theta)\right)^{2}-\varphi_{2(n-1)}^{(\alpha, \alpha)}(\theta) \varphi_{2(n+1)}^{(\alpha, \alpha)}(\theta) \\
& \geq-\frac{(2 \alpha+1)(\sin (2 \theta))^{2}}{(2 n-1)(n+\alpha+1)}\left(\varphi_{2 n}^{(\alpha, \alpha)}(\theta)\right)^{2},
\end{aligned}
$$

with equality only for $\theta \in\left\{0, \frac{\pi}{4}\right\}$.

2) $\forall \theta \in\left[0, \frac{\pi}{4}\right]$,

$$
\left(\varphi_{2 n}^{(\alpha, \alpha)}(\theta)\right)^{2}-\varphi_{2(n-1)}^{(\alpha, \alpha)}(\theta) \varphi_{2(n+1)}^{(\alpha, \alpha)}(\theta) \geq 0
$$

if and only if $-1<\alpha \leq-\frac{1}{2}$.

$$
\begin{aligned}
& \text { 3) } \forall \theta \in\left[0, \frac{\pi}{4}\right], \\
& \frac{(2 n+1)(n+\alpha)}{(2 n-1)(n+\alpha+1)}\left(\varphi_{2 n}^{(\alpha, \alpha)}(\theta)\right)^{2}-\varphi_{2(n-1)}^{(\alpha, \alpha)}(\theta) \varphi_{2(n+1)}^{(\alpha, \alpha)}(\theta) \\
& \geq \frac{2 \alpha+1}{(2 n-1)(n+\alpha+1)}(\cos (2 \theta))^{2}\left(\varphi_{2 n}^{(\alpha, \alpha)}(\theta)\right)^{2},
\end{aligned}
$$

with equality only for $\theta \in\left\{0, \frac{\pi}{4}\right\}$.

$$
\begin{aligned}
& \text { 4) } \forall \theta \in\left[0, \frac{\pi}{4}\right] \text {, } \\
& \varphi_{2(n-1)}^{(\alpha, \alpha)}(\theta) \varphi_{2(n+1)}^{(\alpha, \alpha)}(\theta) \leq \frac{(2 n+1)(n+\alpha)}{(2 n-1)(n+\alpha+1)}\left(\varphi_{2 n}^{(\alpha, \alpha)}(\theta)\right)^{2}
\end{aligned}
$$

if and only if $\alpha \geq-\frac{1}{2}$. 
5) $\forall \theta \in\left[0, \frac{\pi}{4}\right]$,

$$
\begin{aligned}
& \left(\varphi_{2 n+1}^{(\alpha, \alpha)}(\theta)\right)^{2}-\varphi_{2 n-1}^{(\alpha, \alpha)}(\theta) \varphi_{2 n+3}^{(\alpha, \alpha)}(\theta) \\
& \geq-\frac{(2 \alpha-1)(\sin (2 \theta))^{2}}{(2 n+1)(n+\alpha+1)}\left(\varphi_{2 n+1}^{(\alpha, \alpha)}(\theta)\right)^{2}
\end{aligned}
$$

with equality only for $\theta \in\left\{0, \frac{\pi}{4}\right\}$.

$$
\text { 6) } \begin{aligned}
\forall \theta & {\left[0, \frac{\pi}{4}\right], } \\
& \left(\varphi_{2 n+1}^{(\alpha, \alpha)}(\theta)\right)^{2}-\varphi_{2 n-1}^{(\alpha, \alpha)}(\theta) \varphi_{2 n+3}^{(\alpha, \alpha)}(\theta) \geq 0
\end{aligned}
$$

if and only if $-1<\alpha \leq \frac{1}{2}$.

$$
\begin{aligned}
& \text { 7) } \forall \theta \in\left[0, \frac{\pi}{4}\right], \\
& \frac{(2 n+3)(n+\alpha)}{(2 n+1)(n+\alpha+1)}\left(\varphi_{2 n+1}^{(\alpha, \alpha)}(\theta)\right)^{2}-\varphi_{2 n-1}^{(\alpha, \alpha)}(\theta) \varphi_{2 n+3}^{(\alpha, \alpha)}(\theta) \\
& \geq \frac{2 \alpha-1}{(2 n+1)(n+\alpha+1)}(\cos (2 \theta))^{2}\left(\varphi_{2 n+1}^{(\alpha, \alpha)}(\theta)\right)^{2},
\end{aligned}
$$

with equality only for $\theta \in\left\{0, \frac{\pi}{4}\right\}$.

$$
\begin{aligned}
& \text { 8) } \forall \theta \in\left[0, \frac{\pi}{4}\right] \text {, } \\
& \varphi_{2 n-1}^{(\alpha, \alpha)}(\theta) \varphi_{2 n+3}^{(\alpha, \alpha)}(\theta) \leq \frac{(2 n+3)(n+\alpha)}{(2 n+1)(n+\alpha+1)}\left(\varphi_{2 n+1}^{(\alpha, \alpha)}(\theta)\right)^{2}
\end{aligned}
$$

if and only if $\alpha \geq \frac{1}{2}$.

From (10), Theorem 3.4, Corollary 3.5, and Proposition 3.12 , we deduce the following inequalities related to $\mathfrak{I} \psi_{2 m}^{(\alpha, \alpha)}$ :

Proposition 3.16 For all $m \in \mathbb{Z} \backslash\{-1,0,1\}$, we have

$$
\begin{aligned}
& \text { 1) } \forall \theta \in\left[-\frac{\pi}{4}, \frac{\pi}{4}\right], \\
& \left(\mathfrak{\Im} \psi_{2 m}^{(\alpha, \alpha)}(\theta)\right)^{2}-\mathfrak{\Im} \psi_{2(m-1)}^{(\alpha, \alpha)}(\theta) \Im \psi_{2(m+1)}^{(\alpha, \alpha)}(\theta) \\
& \geq\left[-\frac{\lambda_{2(m-1)}^{(\alpha, \alpha)} \lambda_{2(m+1)}^{(\alpha, \alpha)}}{\left(\lambda_{2 m}^{(\alpha, \alpha)}\right)^{2}} \frac{2 \alpha+1}{(2|m|-1)(|m|+\alpha+1)}(\sin (2 \theta))^{2}\right. \\
& \left.+1-\frac{\lambda_{2(m-1)}^{(\alpha, \alpha)} \lambda_{2(m+1)}^{(\alpha, \alpha)}}{\left(\lambda_{2 m}^{(\alpha, \alpha)}\right)^{2}}\right] \times\left(\Im \psi_{2 m}^{(\alpha, \alpha)}(\theta)\right)^{2},
\end{aligned}
$$

with equality only for $\theta \in\left\{-\frac{\pi}{4}, 0, \frac{\pi}{4}\right\}$.

2) $\forall \theta \in\left[-\frac{\pi}{4}, \frac{\pi}{4}\right]$,

$$
\begin{aligned}
& \Im \psi_{2(m-1)}^{(\alpha, \alpha)}(\theta) \Im \psi_{2(m+1)}^{(\alpha, \alpha)}(\theta) \\
\leq & \frac{\lambda_{2(m-1)}^{(\alpha, \alpha)} \lambda_{2(m+1)}^{(\alpha, \alpha)}}{\left(\lambda_{2 m}^{(\alpha, \alpha)}\right)^{2}}\left(\Im \psi_{2 m}^{(\alpha, \alpha)}(\theta)\right)^{2}
\end{aligned}
$$

if and only if $-1<\alpha \leq-\frac{1}{2}$.

3) $\forall \theta \in\left[-\frac{\pi}{4}, \frac{\pi}{4}\right]$,

$$
\begin{aligned}
& \frac{(2|m|+1)(|m|+\alpha)}{(2|m|-1)(|m|+\alpha+1)}\left(\Im \psi_{2 m}^{(\alpha, \alpha)}(\theta)\right)^{2} \\
& -\mathfrak{I} \psi_{2(m-1)}^{(\alpha, \alpha)}(\theta) \mathfrak{I} \psi_{2(m+1)}^{(\alpha, \alpha)}(\theta) \\
& \geq\left[\frac{\lambda_{2(m-1)}^{(\alpha, \alpha)} \lambda_{2(m+1)}^{(\alpha, \alpha)}}{\left(\lambda_{2 m}^{(\alpha, \alpha)}\right)^{2}} \frac{2 \alpha-1}{(2|m|+1)(|m|+\alpha)}(\cos (2 \theta))^{2}\right. \\
& \left.+1-\frac{\lambda_{2(m-1)}^{(\alpha, \alpha)} \lambda_{2(m+1)}^{(\alpha, \alpha)}}{\left(\lambda_{2 m}^{(\alpha, \alpha)}\right)^{2}}\right] \times \frac{(2|m|+1)(|m|+\alpha)}{(2|m|-1)(|m|+\alpha+1)} \\
& \times\left(\Im \psi_{2 m}^{(\alpha, \alpha)}(\theta)\right)^{2}
\end{aligned}
$$

with equality only for $\theta \in\left\{-\frac{\pi}{4}, 0, \frac{\pi}{4}\right\}$.

$$
\begin{aligned}
\text { 4) } \forall \theta \in\left[-\frac{\pi}{4}, \frac{\pi}{4}\right], \\
\text { } \psi_{2(m-1)}^{(\alpha, \alpha)}(\theta) \Im \psi_{2(m+1)}^{(\alpha, \alpha)}(\theta) \\
\leq \frac{\lambda_{2(m-1)}^{(\alpha, \alpha)} \lambda_{2(m+1)}^{(\alpha, \alpha)} \frac{(2|m|+1)(|m|+\alpha)}{(2|m|-1)(|m|+\alpha+1)}\left(\Im \psi_{2 m}^{(\alpha, \alpha)}(\theta)\right)^{2}}{\left(\lambda_{2 m}^{(\alpha, \alpha)}\right)^{2}}
\end{aligned}
$$

if and only if $\alpha \geq-\frac{1}{2}$.

By using (10), Theorem 3.7, Corollary 3.8, and Proposition 3.13, we can show that the Gegenbauer-Dunkl polynomials $\psi_{2 m}^{(\alpha, \alpha)}$ satisfy the following inequalities:

Proposition 3.17 For all $m \in \mathbb{Z} \backslash\{-1,0,1\}$, we have

$$
\begin{aligned}
& \text { 1) } \forall \theta \in\left[-\frac{\pi}{4}, \frac{\pi}{4}\right], \\
& \left|\psi_{2 m}^{(\alpha, \alpha)}(\theta)\right|^{2}-\Re\left(\psi_{2(m-1)}^{(\alpha, \alpha)}(\theta) \overline{\psi_{2(m+1)}^{(\alpha, \alpha)}(\theta)}\right) \\
& \geq-\frac{2 \alpha+1}{(2|m|-1)(|m|+\alpha+1)}(\sin (2 \theta))^{2}\left|\psi_{2 m}^{(\alpha, \alpha)}(\theta)\right|^{2},
\end{aligned}
$$

with equality only for $\theta \in\left\{-\frac{\pi}{4}, 0, \frac{\pi}{4}\right\}$.

$$
\begin{aligned}
& \text { 2) } \forall \theta \in\left[-\frac{\pi}{4}, \frac{\pi}{4}\right], \\
& \left|\psi_{2 m}^{(\alpha, \alpha)}(\theta)\right|^{2}-\Re\left(\psi_{2(m-1)}^{(\alpha, \alpha)}(\theta) \overline{\psi_{2(m+1)}^{(\alpha, \alpha)}(\theta)}\right) \geq 0
\end{aligned}
$$


if and only if $-1<\alpha \leq-\frac{1}{2}$.

$$
\begin{aligned}
& \text { 3) } \forall \theta \in\left[-\frac{\pi}{4}, \frac{\pi}{4}\right], \\
& \frac{(2|m|+1)(|m|+\alpha)}{(2|m|-1)(|m|+\alpha+1)}\left|\psi_{2 m}^{(\alpha, \alpha)}(\theta)\right|^{2} \\
& -\mathfrak{R}\left(\psi_{2(m-1)}^{(\alpha, \alpha)}(\theta) \overline{\psi_{2(m+1)}^{(\alpha, \alpha)}(\theta)}\right) \\
& \geq \frac{2 \alpha-1}{(2|m|-1)(|m|+\alpha+1)}(\cos (2 \theta))^{2}\left|\psi_{2 m}^{(\alpha, \alpha)}(\theta)\right|^{2},
\end{aligned}
$$

with equality only for $\theta \in\left\{-\frac{\pi}{4}, 0, \frac{\pi}{4}\right\}$.

$$
\text { 4) } \begin{aligned}
\forall \theta & \in\left[-\frac{\pi}{4}, \frac{\pi}{4}\right], \\
& \Re\left(\psi_{2(m-1)}^{(\alpha, \alpha)}(\theta) \overline{\psi_{2(m+1)}^{(\alpha, \alpha)}(\theta)}\right) \\
& \leq \frac{(2|m|+1)(|m|+\alpha)}{(2|m|-1)(|m|+\alpha+1)}\left|\psi_{2 m}^{(\alpha, \alpha)}(\theta)\right|^{2}
\end{aligned}
$$

if and only if $\alpha \geq-\frac{1}{2}$.

Also, (10), Theorem 3.9, Corollary 3.10, and Proposition 3.14 give the following proposition:

Proposition 3.18 For all $m \in \mathbb{Z} \backslash\{-1,0,1\}$, we have

$$
\begin{aligned}
& \text { 1) } \forall \theta \in\left[-\frac{\pi}{4}, \frac{\pi}{4}\right], \\
& \left(\Re \psi_{2 m}^{(\alpha, \alpha)}(\theta)\right)^{2}+\frac{\lambda_{2(m-1)}^{(\alpha, \alpha)} \lambda_{2(m+1)}^{(\alpha, \alpha)}}{\left(\lambda_{2 m}^{(\alpha, \alpha)}\right)^{2}}\left(\Im \psi_{2 m}^{(\alpha, \alpha)}(\theta)\right)^{2} \\
& -\Re\left(\psi_{2(m-1)}^{(\alpha, \alpha)}(\theta) \frac{\left.\frac{\left(\psi_{2(m+1)}^{(\alpha, \alpha)}(\theta)\right.}{\alpha}\right)}{\geq-\frac{2 \alpha+1}{(2|m|-1)(|m|+\alpha+1)}(\sin (2 \theta))^{2}}\right. \\
& \times\left[\left(\Re \psi_{2 m}^{(\alpha, \alpha)}(\theta)\right)^{2}+\frac{\lambda_{2(m-1)}^{(\alpha, \alpha)} \lambda_{2(m+1)}^{(\alpha, \alpha)}}{\left(\lambda_{2 m}^{(\alpha, \alpha)}\right)^{2}}\left(\Im \psi_{2 m}^{(\alpha, \alpha)}(\theta)\right)^{2}\right],
\end{aligned}
$$

with equality only for $\theta \in\left\{-\frac{\pi}{4}, 0, \frac{\pi}{4}\right\}$.

$$
\begin{aligned}
& \text { 2) } \forall \theta \in\left[-\frac{\pi}{4}, \frac{\pi}{4}\right], \\
& \Re\left(\psi_{2(m-1)}^{(\alpha, \alpha)}(\theta) \overline{\psi_{2(m+1)}^{(\alpha, \alpha)}(\theta)}\right) \\
& \leq\left(\mathfrak{R} \psi_{2 m}^{(\alpha, \alpha)}(\theta)\right)^{2}+\frac{\lambda_{2(m-1)}^{(\alpha, \alpha)} \lambda_{2(m+1)}^{(\alpha, \alpha)}}{\left(\lambda_{2 m}^{(\alpha, \alpha)}\right)^{2}}\left(\Im \psi_{2 m}^{(\alpha, \alpha)}(\theta)\right)^{2}
\end{aligned}
$$

if and only if $-1<\alpha \leq-\frac{1}{2}$.

$$
\text { 3) } \forall \theta \in\left[-\frac{\pi}{4}, \frac{\pi}{4}\right] \text {, }
$$

$$
\begin{aligned}
& \frac{(2|m|+1)(|m|+\alpha)}{(2|m|-1)(|m|+\alpha+1)} \\
& \times\left[\left(\Re \psi_{2 m}^{(\alpha, \alpha)}(\theta)\right)^{2}+\frac{\lambda_{2(m-1)}^{(\alpha, \alpha)} \lambda_{2(m+1)}^{(\alpha, \alpha)}}{\left(\lambda_{2 m}^{(\alpha, \alpha)}\right)^{2}}\left(\Im \psi_{2 m}^{(\alpha, \alpha)}(\theta)\right)^{2}\right] \\
& -\Re\left(\psi_{2(m-1)}^{(\alpha, \alpha)}(\theta) \overline{\psi_{2(m+1)}^{(\alpha, \alpha)}(\theta)}\right) \\
& \geq \frac{2 \alpha-1}{(2|m|-1)(|m|+\alpha+1)}(\cos (2 \theta))^{2} \\
& \times\left[\left(\Re \psi_{2 m}^{(\alpha, \alpha)}(\theta)\right)^{2}+\frac{\lambda_{2(m-1)}^{(\alpha, \alpha)} \lambda_{2(m+1)}^{(\alpha, \alpha)}}{\left(\lambda_{2 m}^{(\alpha, \alpha)}\right)^{2}}\left(\Im \psi_{2 m}^{(\alpha, \alpha)}(\theta)\right)^{2}\right],
\end{aligned}
$$

with equality only for $\theta \in\left\{-\frac{\pi}{4}, 0, \frac{\pi}{4}\right\}$.

$$
\begin{aligned}
& \text { 4) } \forall \theta \in\left[-\frac{\pi}{4}, \frac{\pi}{4}\right], \\
& \Re\left(\psi_{2(m-1)}^{(\alpha, \alpha)}(\theta) \overline{\psi_{2(m+1)}^{(\alpha, \alpha)}(\theta)}\right) \leq \frac{(2|m|+1)(|m|+\alpha)}{(2|m|-1)(|m|+\alpha+1)}
\end{aligned}
$$

$$
\times\left[\left(\Re \psi_{2 m}^{(\alpha, \alpha)}(\theta)\right)^{2}+\frac{\lambda_{2(m-1)}^{(\alpha, \alpha)} \lambda_{2(m+1)}^{(\alpha, \alpha)}}{\left(\lambda_{2 m}^{(\alpha, \alpha)}\right)^{2}}\left(\Im \psi_{2 m}^{(\alpha, \alpha)}(\theta)\right)^{2}\right]
$$

if and only if $\alpha \geq-\frac{1}{2}$.

\section{Examples 3.19}

1)If we take $\alpha=\beta=\frac{1}{2}$ in Proposition 3.1, one can show that

$$
\forall n \in \mathbb{N}, \forall \theta \in[0, \pi],|\sin (n \theta)| \leq n \sin \theta .
$$

2) If we take $\alpha=-\frac{1}{2}$ and $\beta=\frac{1}{2}$ in Proposition 3.1, one can show that

$$
\forall n \in \mathbb{N}, \forall \theta \in\left[0, \frac{\pi}{2}\right],|\cos ((2 n+1) \theta)| \leq(2 n+1) \cos \theta .
$$

The study of the first terms of the Jacobi-Dunkl polynomials gives the following inequalities:

\section{Proposition 3.20}

$$
\begin{gathered}
\forall m \in\{-1,0,1\}, \forall \theta \in\left[-\frac{\pi}{2}, \frac{\pi}{2}\right], \\
\left(\mathfrak{J} \psi_{m}(\theta)\right)^{2}-\mathfrak{J} \psi_{m-1}(\theta) \mathfrak{J} \psi_{m+1}(\theta) \geq 0,
\end{gathered}
$$

with equality only for $\theta \in\left\{-\frac{\pi}{2}, 0, \frac{\pi}{2}\right\}$.

Proof:

$$
\forall m \in\{-1,0,1\}, \forall \theta \in\left[-\frac{\pi}{2}, \frac{\pi}{2}\right],
$$

$$
\begin{aligned}
& \left(\Im \psi_{m}(\theta)\right)^{2}-\Im \psi_{m-1}(\theta) \Im \psi_{m+1}(\theta)=\left(\Im \psi_{1}(\theta)\right)^{2} \\
& =\frac{\rho+1}{4(\alpha+1)^{2}}(\sin (2 \theta))^{2} \geq 0 .
\end{aligned}
$$


Remark 3.21 The members of the inequality, given in Corollary 3.5 , take the value zero when $m \in\{-1,1\}$, $\alpha>-1$ and $\beta>-1$.

\section{Proposition 3.22}

1) If $-1<\beta \leq \alpha$, then

$$
\forall \theta \in\left[-\frac{\pi}{2}, \frac{\pi}{2}\right],\left|\psi_{0}(\theta)\right|^{2}-\mathfrak{R}\left(\psi_{-1}(\theta) \overline{\psi_{1}(\theta)}\right) \geq 0,
$$

with equality only for $\theta=0$ or $\left(\theta= \pm \frac{\pi}{2}\right.$ and $\left.\beta=\alpha\right)$.

2) If $-1<\alpha<\beta$, let

$$
\theta_{0}:=\arctan \left(\sqrt{\frac{2 \alpha+3}{\beta-\alpha}}\right)
$$

then

a) $\forall \theta \in]-\theta_{0}, \theta_{0}[\backslash\{0\}$,

$$
\mathfrak{R}\left(\psi_{-1}(\theta) \overline{\psi_{1}(\theta)}\right)<\left|\psi_{0}(\theta)\right|^{2} .
$$

b) $\forall \theta \in\left[-\frac{\pi}{2},-\theta_{0}[\cup] \theta_{0}, \frac{\pi}{2}\right]$,

$$
\left|\psi_{0}(\theta)\right|^{2}<\mathfrak{R}\left(\psi_{-1}(\theta) \overline{\psi_{1}(\theta)}\right) .
$$

c) $\forall \theta \in\left\{-\theta_{0}, 0, \theta_{0}\right\}$,

$$
\mathfrak{R}\left(\psi_{-1}(\theta) \overline{\psi_{1}(\theta)}\right)=\left|\psi_{0}(\theta)\right|^{2}=1 .
$$

Proof: $\forall \theta \in\left[-\frac{\pi}{2}, \frac{\pi}{2}\right]$,

$$
\left|\psi_{0}(\theta)\right|^{2}-\mathfrak{R}\left(\psi_{-1}(\theta) \overline{\psi_{1}(\theta)}\right)
$$$$
=\frac{\rho+1}{4(\alpha+1)^{2}}(\sin (2 \theta))^{2}+1-\left(\varphi_{1}(\theta)\right)^{2}
$$$$
=\frac{(\rho+1)}{(\alpha+1)^{2}}(\sin \theta)^{2}\left[(2 \alpha+3)(\cos \theta)^{2}+(\alpha-\beta)(\sin \theta)^{2}\right] \text {. }
$$

The derivative of the function

$$
f: \theta \mapsto(2 \alpha+3)(\cos \theta)^{2}+(\alpha-\beta)(\sin \theta)^{2}
$$

is $f^{\prime}: \theta \mapsto-(\rho+2) \sin (2 \theta)$. Then $\left.\forall \theta \in\right] 0, \frac{\pi}{2}\left[, \quad f^{\prime}(\theta)<0\right.$.

Moreover, $f(0)=2 \alpha+3>0$ and $f\left(\frac{\pi}{2}\right)=\alpha-\beta<0$ when $-1<\alpha<\beta$. Thus we get the sign of $f(\theta)$ which finishes the proof.

\section{Corollary 3.23}

1) If $-1<\beta \leq \alpha$, then

$$
\forall \theta \in\left[0, \frac{\pi}{2}\right], \quad\left|\varphi_{1}(\theta)\right| \leq\left(\frac{\rho+1}{4(\alpha+1)^{2}}(\sin (2 \theta))^{2}+1\right)^{\frac{1}{2}},
$$

with equality only for $\theta=0$ or $\left(\theta=\frac{\pi}{2}\right.$ and $\left.\beta=\alpha\right)$.

$$
\text { 2) If }-1<\alpha<\beta \text {, then }
$$

a) $\forall \theta \in] 0, \theta_{0}[$,

$$
\left|\varphi_{1}(\theta)\right|<\left(\frac{\rho+1}{4(\alpha+1)^{2}}(\sin (2 \theta))^{2}+1\right)^{\frac{1}{2}} .
$$

b) $\left.\forall \theta \in] \theta_{0}, \frac{\pi}{2}\right]$,

$$
\left(\frac{\rho+1}{4(\alpha+1)^{2}}(\sin (2 \theta))^{2}+1\right)^{\frac{1}{2}}<\left|\varphi_{1}(\theta)\right| \text {. }
$$

c) $\forall \theta \in\left\{0, \theta_{0}\right\}$,

$$
\left|\varphi_{1}(\theta)\right|=\left(\frac{\rho+1}{4(\alpha+1)^{2}}(\sin (2 \theta))^{2}+1\right)^{\frac{1}{2}}=1,
$$

where $\theta_{0}$ is given by (13).

Proposition 3.24 Let $m \in\{-1,1\}$.

1) If $-1<\alpha \leq \beta$, then $\forall \theta \in\left[-\frac{\pi}{2}, \frac{\pi}{2}\right], \quad\left(\Re \psi_{m}(\theta)\right)^{2}-\mathfrak{R}\left(\psi_{m-1}(\theta) \overline{\psi_{m+1}(\theta)}\right) \geq 0$, with equality only for $\theta=0$ or $\left(\theta= \pm \frac{\pi}{2}\right.$ and $\left.\beta=\alpha\right)$.

2) If $-1<\beta<\alpha$, let

$$
\theta_{1}:=\arctan \left(\sqrt{\frac{2(\alpha+1)(\alpha+2)}{(\alpha-\beta)(\beta+1)}}\right),
$$

then

a) $\forall \theta \in]-\theta_{1}, \theta_{1}[\backslash\{0\}$,

$$
\mathfrak{R}\left(\psi_{m-1}(\theta) \overline{\psi_{m+1}(\theta)}\right)<\left(\mathfrak{R} \psi_{m}(\theta)\right)^{2} .
$$

b) $\forall \theta \in\left[-\frac{\pi}{2},-\theta_{1}[\cup] \theta_{1}, \frac{\pi}{2}\right]$,

$$
\left(\Re \psi_{m}(\theta)\right)^{2}<\mathfrak{R}\left(\psi_{m-1}(\theta) \overline{\psi_{m+1}(\theta)}\right) .
$$

c) $\forall \theta \in\left\{-\theta_{1}, 0, \theta_{1}\right\}$,

$$
\mathfrak{R}\left(\psi_{m-1}(\theta) \overline{\psi_{m+1}(\theta)}\right)=\left(\mathfrak{R} \psi_{m}(\theta)\right)^{2} .
$$

Proof: $\forall \theta \in\left[-\frac{\pi}{2}, \frac{\pi}{2}\right]$,

$$
\begin{aligned}
& \mathfrak{R}\left(\psi_{m-1}(\theta) \overline{\psi_{m+1}(\theta)}\right)-\left(\mathfrak{R} \psi_{m}(\theta)\right)^{2} \\
& =\varphi_{2}(\theta)-\left(\varphi_{1}(\theta)\right)^{2} \\
& =-\frac{(\sin \theta)^{2}}{(\alpha+1)^{2}(\alpha+2)} \\
& \times\left[2(\alpha+1)(\alpha+2)(\cos \theta)^{2}+(\beta+1)(\beta-\alpha)(\sin \theta)^{2}\right] .
\end{aligned}
$$

The derivative of the function $g: \theta \mapsto 2(\alpha+1)(\alpha+2)(\cos \theta)^{2}+(\beta+1)(\beta-\alpha)(\sin \theta)^{2}$ 
is $\quad g^{\prime}: \theta \mapsto[(\beta+1)(\beta-\alpha)-2(\alpha+1)(\alpha+2)] \sin (2 \theta)$. If $-1<\beta<\alpha$, then $\forall \theta \in] 0, \frac{\pi}{2}\left[, g^{\prime}(\theta)<0\right.$. . Moreover, $g(0)=2(\alpha+1)(\alpha+2)>0$ and $g\left(\frac{\pi}{2}\right)=(\beta+1)(\beta-\alpha)<0$.

Thus we get the sign of $g(\theta)$ which finishes the proof.

\section{Remark 3.25}

$$
\begin{aligned}
& \forall m \in \mathbb{Z}, \\
& \mathfrak{R}\left(\psi_{m-1}(0) \overline{\psi_{m+1}(0)}\right)=\left(\mathfrak{R} \psi_{m}(0)\right)^{2} \\
& =\left|\psi_{m}(0)\right|^{2}=1 .
\end{aligned}
$$

\section{Corollary 3.26}

1) If $-1<\alpha \leq \beta$, then

$$
\forall \theta \in\left[0, \frac{\pi}{2}\right], \varphi_{2}(\theta) \leq\left(\varphi_{1}(\theta)\right)^{2},
$$

with equality only for $\theta=0$ or $\left(\theta=\frac{\pi}{2}\right.$ and $\left.\beta=\alpha\right)$.

2) If $-1<\beta<\alpha$, then

a) $\forall \theta \in] 0, \theta_{1}\left[, \varphi_{2}(\theta)<\left(\varphi_{1}(\theta)\right)^{2}\right.$.

b) $\left.\forall \theta \in] \theta_{1}, \frac{\pi}{2}\right],\left(\varphi_{1}(\theta)\right)^{2}<\varphi_{2}(\theta)$.

c) $\forall \theta \in\left\{0, \theta_{1}\right\}, \varphi_{2}(\theta)=\left(\varphi_{1}(\theta)\right)^{2}$, where $\theta_{1}$ is given by (14).

Proposition 3.27 Let $m \in\{-1,1\}$.

1) If $-1<\alpha \leq \beta$, then

$$
\forall \theta \in\left[-\frac{\pi}{2}, \frac{\pi}{2}\right],\left|\psi_{m}(\theta)\right|^{2}-\mathfrak{R}\left(\psi_{m-1}(\theta) \overline{\psi_{m+1}(\theta)}\right) \geq 0,
$$

with equality only for $\theta=0$ or $\left(\theta= \pm \frac{\pi}{2}\right.$ and $\left.\beta=\alpha\right)$.

2) If $-1<\beta<\alpha$, let

$$
\theta_{2}=\arctan \left(\sqrt{\frac{(\alpha+2)(3 \alpha+\beta+4)}{(\alpha-\beta)(\beta+1)}}\right)
$$

then

a) $\forall \theta \in]-\theta_{2}, \theta_{2}[\backslash\{0\}$,

$$
\mathfrak{R}\left(\psi_{m-1}(\theta) \overline{\psi_{m+1}(\theta)}\right)<\left|\psi_{m}(\theta)\right|^{2} .
$$

b) $\forall \theta \in\left[-\frac{\pi}{2},-\theta_{2}[\cup] \theta_{2}, \frac{\pi}{2}\right]$,

$$
\left|\psi_{m}(\theta)\right|^{2}<\mathfrak{R}\left(\psi_{m-1}(\theta) \overline{\psi_{m+1}(\theta)}\right) .
$$

c) $\forall \theta \in\left\{-\theta_{2}, 0, \theta_{2}\right\}$,

$$
\mathfrak{R}\left(\psi_{m-1}(\theta) \overline{\psi_{m+1}(\theta)}\right)=\left|\psi_{m}(\theta)\right|^{2} .
$$

Proof:

$$
\forall \theta \in\left[-\frac{\pi}{2}, \frac{\pi}{2}\right], \quad\left|\psi_{m}(\theta)\right|^{2}-\mathfrak{R}\left(\psi_{m-1}(\theta) \overline{\psi_{m+1}(\theta)}\right)
$$

$$
\begin{aligned}
& =\left|\psi_{1}(\theta)\right|^{2}-\varphi_{2}(\theta) \\
& =\left(\varphi_{1}(\theta)\right)^{2}+\frac{\rho+1}{4(\alpha+1)^{2}}(\sin (2 \theta))^{2}-\varphi_{2}(\theta) \\
& =\frac{(\sin \theta)^{2}}{(\alpha+1)^{2}(\alpha+2)} \\
& \times\left[(\alpha+2)(3 \alpha+\beta+4)(\cos \theta)^{2}+(\beta+1)(\beta-\alpha)(\sin \theta)^{2}\right] .
\end{aligned}
$$

The derivative of the function

$h: \theta \mapsto(\alpha+2)(3 \alpha+\beta+4)(\cos \theta)^{2}+(\beta+1)(\beta-\alpha)(\sin \theta)^{2}$.

is $h^{\prime}: \theta \mapsto[(\beta+1)(\beta-\alpha)-(\alpha+2)(3 \alpha+\beta+4)] \sin (2 \theta)$. If $-1<\beta<\alpha$, then $\forall \theta \in] 0, \frac{\pi}{2}\left[, h^{\prime}(\theta)<0\right.$. Moreover, $h(0)=(\alpha+2)(3 \alpha+\beta+4)>0$ and $h\left(\frac{\pi}{2}\right)=(\beta+1)(\beta-\alpha)<0$

Thus we get the sign of $h(\theta)$ which finishes the proof.

\section{Corollary 3.28}

1) If $-1<\alpha \leq \beta$, then

$$
\forall \theta \in\left[0, \frac{\pi}{2}\right], \varphi_{2}(\theta) \leq\left(\varphi_{1}(\theta)\right)^{2}+\frac{\rho+1}{4(\alpha+1)^{2}}(\sin (2 \theta))^{2},
$$

with equality only for $\theta=0$ or $\left(\theta=\frac{\pi}{2}\right.$ and $\left.\beta=\alpha\right)$.

2) If $-1<\beta<\alpha$, then

a) $\forall \theta \in] 0, \theta_{2}[$,

$$
\varphi_{2}(\theta)<\left(\varphi_{1}(\theta)\right)^{2}+\frac{\rho+1}{4(\alpha+1)^{2}}(\sin (2 \theta))^{2} .
$$

b) $\left.\forall \theta \in] \theta_{2}, \frac{\pi}{2}\right]$,

$$
\left(\varphi_{1}(\theta)\right)^{2}+\frac{\rho+1}{4(\alpha+1)^{2}}(\sin (2 \theta))^{2}<\varphi_{2}(\theta) .
$$

c) $\forall \theta \in\left\{0, \theta_{2}\right\}$,

$$
\varphi_{2}(\theta)=\left(\varphi_{1}(\theta)\right)^{2}+\frac{\rho+1}{4(\alpha+1)^{2}}(\sin (2 \theta))^{2},
$$

where $\theta_{2}$ is given by (15).

\section{Acknowledgements}

Thanks to the referee for careful reading and helpful comments.

\section{References}

[1] Gasper, G., An inequality of Turán type for Jacobi polynomials, Proc. Amer. Math. Soc., 32(2). 435-439. 1972.

[2] Turán, P., On the zeros of the polynomials of Legendre, Časopis. Pěst. Mat. Fys., 75. 113-122. 1950.

[3] Szegö, G., On an inequality of P. Turán concerning Legendre polynomials, Bull. Amer. Math. Soc., 54. 401-405. 1948. 
[4] Alzer, H., Gerhold, S., Kauers, M. and Lupas, A., On Turán's inequality for Legendre polynomials, Expo. Math., 25. 181-186. 2007.

[5] Baricz, Á., Turán type inequalities for hypergeometric functions, Proc. Amer. Math. Soc., 136(9). 3223-3229. 2008.

[6] Bustoz, J. and Savage, N., Inequalities for ultraspherical and Laguerre polynomials, SIAM J. Math. Anal., 10(5). 902-912. 1979.

[7] Bustoz, J. and Ismail, M.E.H., Turán inequalities for ultraspherical and continuous q-ultraspherical polynomials. SIAM J. Math. Anal., 14. 807-818. 1983.

[8] Bustoz, J. and Ismail, M.E.H., Turán inequalities for symmetric orthogonal polynomials, Internat. J. Math. Math. Sci., 20(1). 1-8. 1997.

[9] Bustoz, J. and Pyung, I.S., Determinant inequalities for sieved ultraspherical polynomials, Internat. J. Math. Math. Sci., 25(11). 745-751. 2001.

[10] Gasper, G., On the extension of Turán inequality for Jacobi polynomials. Duke Math. J., 38. 415-428. 1971.

[11] Simic, S., Turán's inequality for Appell polynomials, J. Inequal. Appl., 1-7. 2006.

[12] Szasz, O., Inequalities concerning ultraspherical polynomials and Bessel functions, Proc. Amer. Math. Soc., 1. 256-267. 1950.

[13] Csordas G. and Williamson, J., On polynomials satisfying a Turán type inequality. Proc. Amer. Math. Soc., 43(2). 367-372. 1974.
[14] Danese, A., Some identities and inequalities involving ultraspherical polynomials. Duke Math. J. 26. 349-359. 1959.

[15] Dimitrov, D.K., Higher order Turán inequalities. Proc. Amer. Math. Soc., 126. 2033-2037. 1998

[16] Szegö, G., An inequality for Jacobi polynomials, Studies in Mathematical Analysis and Related Topics, Stanford Univ. Press., Stanford, California, 392-398. 1962.

[17] Venkatachaliengar, K. and Lakshmana Rao, S.K., On Turán's inequality for ultraspherical polynomials, Proc. Amer. Math. Soc., 8. 1075-1087. 1957

[18] Luke, Y.L., The special functions and their approximations, Vol. 2, Academic Press, 1969.

[19] Szegö, G., Orthogonal polynomials, Amer. Math. Soc. Colloq. Pub., Vol. 23, Amer. Math. Soc., Providence, R. I., 1967.

[20] Erdélyi, A., Magnus, W., Oberhettinger, F. and Tricomi, G., Higher transcendental functions, Vol. 1, McGraw-Hill Book Company, 1953.

[21] Luke, Y.L., The special functions and their approximations, Vol. 1, Academic Press, 1969.

[22] Chouchene, F., Harmonic analysis associated with the Jacobi-Dunkl operator on $]-\frac{\pi}{2}, \frac{\pi}{2}[$. J. Comput. Appl. Math., 178. 75-89. 2005. 